\title{
Some kind of relative clause ${ }^{1}$ Jon Ander MENDIA — Heinrich Heine University Düsseldorf
}

\begin{abstract}
Amount Relatives (ARs) differ from restrictive relative clauses in that they do not refer to a particular object denoted by the head of the relative clause, but to an amount of such objects (Carlson, 1977a; Heim, 1987). Traditionally, ARs have been regarded as degree expressions. In this paper I argue against this view and propose instead that amount interpretations of relative clauses are in fact a special case of kind interpretation.
\end{abstract}

Keywords: kind reference, amounts, relative clauses.

\section{Introduction}

This is paper is concerned with Amount Relatives in English, relative clauses that receive quantity-oriented interpretations (Carlson, 1977a; Heim, 1987; Grosu and Landman, 1998, 2017; Herdan, 2008; McNally, 2008; Meier, 2015). Consider the following example, minimally adapted from Heim (1987: 38).

(1) It would take us years to drink the champagne they spilled that evening.

The sentence in (1) is ambiguous. On its ordinary interpretation, the relative clause picks out the particular champagne that was spilled that evening, and the sentence on the whole is about the time it will take to drink that spilled champagne. This is the meaning we arrive at when, following the traditional analysis (Quine, 1960; Partee, 1973), we interpret the relative clause by intersecting the predicate denoted by the head noun with the extension of the that-clause. But this is not the most accessible interpretation of the sentence. On its most salient reading, (1) refers to the task of drinking the amount of champagne that was spilled that evening. In this case, the particular champagne that was spilled is not the object of the drinking; rather any champagne in the same amount will suffice. The examples below provide similar cases. Under the relevant interpretation, they all make a claim about an amount, not about an object.

(2) a. Mary saw the birds in thirty minutes that John saw in a day.

(Meier, 2015) $\leadsto$ Mary saw the number of birds that John saw

b. We lost the battle because we lacked the soldiers our enemy had. (McNally, 2008) $\leadsto$ We lacked the amount of soldiers that our enemy had

c. The money it cost could have fed many people. (Grosu and Landman, 2017) $\leadsto$ The amount of money it cost

Because of their semantic ability to refer to amounts, these relative clauses were named "Amount Relatives" by Carlson (1977a); I will refer to them as ARs henceforth.

\footnotetext{
${ }^{1}$ Many thanks to Rajesh Bhatt and Vincent Homer for the many discussions we had on this and related topics. I would also like to thank Daniel Altshuler, Athulya Aravind, Seth Cable, Elena Castroviejo, Barbara Partee and audiences at UMass Amherst, HHU Düsseldorf and SuB 22 for their insightful comments.
} 
Historically, analyses of ARs have assumed that degree semantics should be invoked, in some form or other, in order to derive their amount interpretations. Picking up on Carlson's idea that the work of extracting an amount should be done at the CP level, the received view has it that in ARs the embedded CP is a degree expression, denoting either a set of degrees or a maximalized degree (Heim, 1987; von Fintel, 1999; Grosu and Landman, 1998, 2017; Herdan, 2008; Meier, 2015). That is, the amount interpretation is the result of some "amount construction", according to which the $\mathrm{CP}$ in (3) should be treated as a degree predicate-involving degree abstraction at the CP level. For instance:

(3) It would take us years to drink the champagne [ ${ }_{\mathrm{CP}}$ they spilled that evening] $\llbracket \mathrm{CP} \rrbracket=\lambda d$. they spilled $d$-MUCH champagne that evening

The CP being degree denoting, these analyses come with the consequence that ARs should show all the properties of other bona fide degree constructions, such as comparatives, equatives, degree questions, etc.

In this paper I provide a radically different approach to ARs. First, I show that there are a number of parallelisms between ARs and relative clauses with kind interpretations, which are unexpected on a degree-based account of ARs. Then, I bring a series of arguments against a degree-based analysis of ARs: contrary to what is expected, ARs do not show any of the hallmark features of well-established degree constructions. From these two sets of facts I conclude in favor of a uniform analysis of kind and amount relatives and against a degree-based approach to their semantics. In the proposal I will advance, amount interpretations are a form of kind interpretation. Consequently, whenever a relative clause admits an amount interpretation, it also necessarily allows a kind interpretation. This is captured by the following generalization:

The AMOUNT $\subseteq$ KIND generalization:

Amount interpretations of relative clauses are a form of kind interpretation.

\section{Amount Relatives in perspective}

\subsection{Main properties of ARs}

There are three main semantic properties of amount interpretations that set them apart from ordinary object-referring (intersective) interpretations. The first and most obvious is that they refer to amounts, not objects. This poses a general challenge: in spite of being DPs of the form the NPs, amount interpretations do not refer to that NP. The flip-side of this property is that the NPs heading the relative clause in (1) and (2) cannot be interpreted as definites, but as indefinites, in spite of the presence of the definite article. For instance, in (1) there is no single individual object-level champagne that would take us long to drink; in fact, any champagne in the relevant amount suffices. The last distinguishing property of amount interpretations is that they always involve a comparison of two amounts of the same stuff. To appreciate this requirement better, consider first a classifier relative clause with an overt noun amount. 
(5) It would take us years to drink the amount of champagne that you drank of wine.

What (5) shows is that relative clauses headed by the noun amount allow the comparison of two different instances of stuff; in (5) the comparison is between amounts champagne and wine. The same, however, is not possible with ARs.

(6) *It would take us years to drink the champagne that you drank wine.

To ensure that this restriction is not syntactic, we can use sentences that are grammatical but lack an amount interpretation in the relevant environments. Consider (7):

(7) [Context: I drank two liters of champagne in 3 hours, and you drank two liters of wine in 30 minutes.]

It took me 3 hours to drink the champagne that you drank in 30 minutes.

Despite its grammaticality and the supportive context, (7) would be deemed false in this situation. This points out that, unlike for (5), comparing amounts of different stuff is not possible for relative clauses like (1) and (7).

Summing up, any theory that aims at accounting for amount interpretations of relative clauses should capture these three empirical facts, summarized in (8) and paraphrased in (9) for clarity:
a. Definiteness:
Amount interpretations refer to a definite amount, not a definite individual.
b. Indefiniteness:
The head of the relative clause is interpreted as an indefinite.
c. Identity:

Amount interpretations require a comparison of two amounts of the same stuff.

(9) 【It would take us years to drink the champagne they spilled that evening $\rrbracket \quad[=(1)]$ $\leftrightarrow$ It would take us years to drink champagne in that amount [where that amount = the amount of champagne that they spilled that evening]

\subsection{A notable parallelism}

The properties of amount interpretations discussed above have been known since Carlson (1977a, b). Carlson also noted that relative clauses allowing for amount interpretations can be kind-referring as well. Consider a minimal variant of (1):

$$
\text { It will take us the rest of our lives to find the champagne they spilled that evening. }
$$

On its most salient interpretation, (10) receives a form of kind interpretation-biased by the change of verb from drink to find. For instance, it could be that the champagne is difficult to find because it is very rare. This is only one of many possible reasons; it could also be that 
there is a high demand of that particular kind of champagne, that it is not usually imported to our country, etc. ${ }^{2}$ All these interpretations have the same properties of amount interpretations described in (8). For one, the sentence does not refer to a particular champagne, despite being overtly of the form the champagne that.... This is precisely the condition on indefiniteness of the head of the relative clause described in (8). Similarly, the sentence refers to a definite kind of champagne, the precise kind of champagne that they spilled that evening. This is, again, fully parallel to the condition on definiteness described in (8). Finally, notice that we are not at liberty to choose what is the thing that would take us the rest of our lives to find; it must be champagne. This is the same identity restriction that we observed in (8) for amount interpretations. To appreciate the parallelism between kind and amount interpretations in full, consider the following equivalent of the paraphrase in (9).

(11) 【It would take us years to drink the champagne that they spilled that evening】

$\leftrightarrow$ It would take us years to drink champagne of that kind

[where that kind $=$ the kind of champagne that they spilled that evening]

The conclusion is clear: the facts in (8) are not exclusive of amount interpretations alone.

\subsection{Further similarities}

There a number of further parallelisms between kind and amount interpretations of relative clauses, suggesting that the connection between the two is not spurious. First, both kind and amount interpretations seem to be a particularity of the definite article.

It would take us years to $\{$ find/drink $\}\left\{\right.$ the $\left./ *_{\text {a }} / *_{\text {some }} / *_{\text {few }} / *_{\text {two }}\right\}$ champagne that they spilled that evening.

Second, the two interpretations are generally incompatible with the complementizer which:

It would take us years to $\{$ find/drink $\}$ the champagne $\{$ that $/ \varnothing / *$ which $\}$ they spilled that evening.

Third, amount interpretations of relative clauses do not obligatorily rely on the presence of a relative clause, and with sufficient contextual support, it may be dropped altogether. Thus, both amount and kind interpretations are possible with nouns modified by PPs.

a. We lost the battle because we didn't have the soldiers of the Imperial Army.

b. We used to organize a soccer team, but we don't have the students in the department anymore.

\footnotetext{
${ }^{2}$ This type of interpretation is sometimes referred to as an "extent" reading, which goes beyond what we usually think of taxonomic kinds. I will continue to call it simply a "kind" interpretation for consistency, but it should be clear that this interpretation is in fact richer than a well-established kind.
} 
For instance, the sentence (14a) might refer to the fact that the reason for losing the battle was that we did not have as many soldiers as the Imperial Army did. This is the amount interpretation. Alternatively, it could be that despite having more soldiers than the Imperial Army, ours are poorly trained, lack motivation, etc. This is the kind interpretation (see fn.2). Similar interpretations are available with bare DPs as well.

a. We lost the battle because we didn't have the soldiers.

b. We used to organize a soccer team, but don't have the students anymore.

Thus, in all three cases we observe that the relationship between the availability of the two interpretations is preserved. More importantly, the presence of amount interpretations in (14) and (15) is puzzling from a perspective where they require a degree variable originating in a subordinate position, as is usually assumed with ARs.

The upshot of this discussion is that we have two types of interpretation, kind and amount, showing the same signature effects, and similar syntactic constraints, distribution and availability. These parallelisms and the fact that kind-reference typically does not involve degrees raise the question: Are we justified in appealing to degree semantics to account for amount interpretations? Minimally, in doing so we would miss a generalization, namely, that amount and kind interpretations of relative clauses share the key semantic properties that make ARs stand out and behave unlike intersective relative clauses. In what follows, I show that not only does appealing to degrees miss a generalization, it also makes the wrong empirical predictions.

\section{Doing without degrees}

This section examines whether so-called ARs behave as bona fide degree constructions. The results of this examination have already been foreshadowed before: if we take comparatives, equatives, etc. as the quintessential degree constructions involving a relative clause and degree abstraction at the CP level, amount interpretations of relative clauses do not behave alike.

\subsection{Sub-deletion}

The process known as sub-deletion is considered a hallmark of degree abstraction (Kennedy, 1999; Lechner, 2001). For instance, comparatives and equatives all allow sub-deletion.

a. I brought more bananas than you brought apples.

b. I brought as many bananas as you brought apples.

Classifier Relatives also allow sub-deletion. In contrast, relative clauses with amount interpretations never allow sub-deletion.

a. I brought the $\{$ amount / quantity $\}$ of bananas that you brought of apples.

b. *It will take us years to drink the champagne that they spilled wine that evening. 
The lack of sub-deletion properties of (17b) points towards a fundamental difference in how the amount interpretations arise in (16)/(17a) and (1)/(2). Thus, we fail to find the expected parallelism between canonical degree constructions and ARs.

\subsection{Islands}

The second argument is the lack of island effects with relative clauses that permit an amount interpretation. There is a subset of syntactic islands, the so-called weak or sensitive islands, which only allow extraction of certain grammatical expressions: expressions ranging over individual entities are good extractees, as opposed to expressions ranging over other domains, like degrees, times, manners, etc., which often incur in so-called island violations. If relative clauses require degree abstraction to obtain amount interpretations, they should pattern together with other constructions that involve the same operation in showing weak-island sensitivity, much like comparatives, equatives and how many questions. By the same token, relative clauses with an amount interpretation should contrast with individual who questions, which involve abstraction over individuals, and are able to be extracted from weak islands.

Below, I examine the behavior of $e$-denoting $v s . d$-denoting $w h$-words in negative islands as a baseline, and compare this with the behavior of comparatives, equatives and relative clauses. ${ }^{3}$ The interaction between degree operators and negative and other downward entailing operators was noted early on the works that pioneered degree semantics for the study of comparative constructions (see von Stechow, 1984). An influential view popularized by Rullmann (1995) attributes the ill-formedness of the (18) examples below to the impossibility of maximalizing a set of degrees that contains a negative operator in its scope.
a. *How many soldiers doesn't the Imperial Army have?
b. *We have more soldiers than the Imperial Army doesn't have.
c. *We have as many soldiers as the Imperial Army doesn't have.

The ungrammaticality of the previous examples contrasts with the grammaticality of cases where the extractee lives in the domain of individuals, such as which and what.

Which soldiers doesn't the Imperial Army have?

If we look at relative clauses with amount interpretations, we observe that they pattern like (19) and unlike the examples in (18) above. Many speakers readily admit an amount reading of (20): it amounts to saying that our soldiers exceeded in number those of the Imperial Army. ${ }^{4}$ (As expected, out of the blue, the kind interpretation of (20) is also available.)

\footnotetext{
${ }^{3}$ Due to space limitations, I only discuss the case of negative islands, but the same observations hold of others, such as tenseless $w h$-islands, a variety of factive constructions, etc.

${ }^{4}$ Some speakers may need some more contextual support. Suppose that our school is competing against others to get some fellowship. In order to get the fellowship there are certain stringent constraints on how many students schools may have, such that having a certain number of students may maximize your chances of obtaining the fellowship. In this case, a sentence like our school got the fellowship because we had the students that yours didn't have expresses that we had an amount of students such that your school did not have as many students.
} 
We won the battle because we had the soldiers that the Imperial Army didn't have.

\subsection{Interim conclusion}

In the last two sections, I demonstrated both that $(i)$ there is an undeniably similarity between amount relatives and kind-referring relatives and (ii) unexpected differences between amount relatives and other degree constructions. These facts suggest that a unified treatment of kind and amount relatives is not just defensible, but desirable. Therefore, I propose the generalization in (4), where amount readings are ultimately derived from kind readings.

(4) The AMOUNT $\subseteq$ KIND generalization:

Amount interpretations of relative clauses are a form of kind interpretation.

\section{What kinds and amounts have in common}

The goal of this section is to spell out a formal account of the generalization in (4). The general intuition that I will pursue, in a nutshell, the following. The relative clauses we have discussed so far make reference to subkinds. The head noun of the relative clause provides the name of a kind that we can then reference and attribute properties to. The kind interpretations prompted by relative clauses in (1) and (2) highlight some relevant property that holds of the referent of the relative clause. This property is used to attribute to the kind-referring term the sufficiently regular behavior that it requires to be understood as kind-referring. Paraphrasing:

\footnotetext{
a. It would take us years to drink the champagne that they spilled last night.

$\sim$ It would take us years to drink champagne with some relevant property of the champagne we spilled last night

b. $\quad[$ DP the champagne that we spilled last night ] $\leadsto$ champagne with property $\mathcal{P}$ [where "the champagne that we spilled last night" is a realization of $\mathcal{P}$ ]
}

Since the relevant property $\mathcal{P}$ that serves to single out the referred kind is unspecified, it could be anything that is supported by the current circumstances, and so it may well be a gradable property like be d-dry, and be d-much, as well a non-gradable property, like be produced in Alsace, or a more common taxonomic property of champagne-kinds, like be a prestige cuvée. In this way it is possible to capture amount interpretations of relative clauses like (1)/(2) by the same means required to account for kind reference. It is in this sense that amount interpretations can be taken to be a form of kind interpretation and that a unified account is possible. As a consequence, whenever a relative clause admits an amount interpretation it also necessarily allows a kind interpretation. This way of looking at sentences like (1)/(2) captures their overall vagueness-i.e. the champagne that was spilled could have any number of properties bearing on the time it would take us to drink an equivalent champagne. The key unifying factor, however, is that the property $\mathcal{P}$ contributes a way of narrowing down the space of possibilities for the subkind in question. 


\subsection{On kinds and subkinds}

Although definite DPs cannot typically be used to form generic statements in English, there are specific environments where the definite article can be used to make reference to a kind. Consider (22), where a kind-referring term is further restricted by the use of an anaphoric demonstrative or a relative clause. In these examples, the head noun "kind" is optional, suggesting that the definite article is not altogether ruled out from kind-referring terms.
a. This (kind of) lion is widespread.
b. This (kind of) whale is extinct.
c. The (kind of) lion that eats people is widespread.
d. The (kind of) whale that had horns is extinct.

Crucially, the sentences in (22) refer to subkinds of lions and whales, as opposed to the natural kinds on the whole. Moreover, subkind-referring expressions like those in (22) need not be natural or well-established; they can be ad-hoc. This is easily seen in (22c): the lions that eat people, for instance, do not form a natural class; in fact, they may comprise of individual lions in several subspecies of lion and exclude others in the same subspecies.

Chierchia (1998) thought of kinds as regularities that occur in nature, whose only property is that "we can impute to them a sufficiently regular behavior". Ad hoc subkinds allow us to do something similar in real time, that is, impute a regular behavior to some subset of a kind without prior agreement as to whether the behavior in question actually qualifies as sufficiently regular. This is a very useful mechanism if, with Chierchia, we believe that what counts as kind is not set by the grammar, but amounts instead to conventional (shared) knowledge of a community of speakers. It allows us to talk and ask questions about very specific kinds. These examples help to pinpoint what we need to form an ad hoc subkind: ( $i$ ) a semantic sortal-something to be a kind of-, and (ii) some means to identify what the relevant subkind is. $(i)$ is provided by a kind-referring noun. Anaphoric demonstratives, relative clauses (and sometimes PPs and other modifiers like adjectives) can accomplish (ii). The analysis I defend here capitalizes on the possibility of constructing ad hoc subkinds and the grammaticality of the definite article when making reference to such subkinds.

\subsection{Ad hoc subkinds as partitions}

Referencing subkinds, ad hoc or not, is not completely free. Carlson (1977a) noted that when referring to different subkinds, the subkinds must be disjoint, they cannot share realizations. A sentence like (23) cannot be verified by a situation where only Fido is sitting in the next room, even though Fido in fact belongs to more than one kind of dog (e.g. if he is a watch dog and a border collie in the real world, he effectively belongs to two different subkinds of dog).

Two kinds of dogs are sitting in the next room.

Carlson (1977b: 213) spelled out the constraint as follows (slightly adapted here): 
Disjointness Condition: A kind-referring expression can only refer to a contextually defined subset of all the possible subkinds that the noun is true of, such that:

a. the subkinds in this subset are disjoint and share no realizations,

b. the subkinds collectively cover all the space of realizations of the kind.

In order to make the connection between ad hoc subkinds and amount interpretations maximally salient, I will recast Carlson's (1977b) disjointness condition in terms of partitions. I suggest that reference to subkinds must be mediated by an equivalence relation that induces a partition on the denotation of its relevant superkind. How this equivalence relation is determined is context dependent; as a consequence, part of the task when interpreting an ad hoc subkind referring expression involves retrieving this equivalence relation from the context.

Following Cresswell (1976), Klein (1980) and many others, degrees can be understood as equivalence classes of ordinary objects. That is, the degree of my height can be defined by the set things that are the same height as me, an amount of champagne as the set of portions of liquid of equal volume, etc. Because interpreting ad hoc subkinds involves figuring out what the equivalence relations are, and because some equivalence relations can serve to define degrees, there is no reason why ad hoc subkinds should not make reference to portions of equal amounts, just like they can refer to sets of entities (qua kinds). Coming back to the example in (21) above, we could say that the equivalence relation be the same kind as would give us a partition of champagne individuals according to their kind (e.g. blanc de noirs, blanc de blancs, rosé champagne...). The equivalence relation be as sweet as would partition the domain of champagne in terms of the sweetness of its instances (extra brut, brut, extra dry...), whereas an equivalence relation be as much as would partition the denotation of champagne in different amounts $(1 L, 2 L, 3 L \ldots$ or perhaps 1 bottle, 2 bottles, 3 bottles...).

Let us look first into partitions. A partition is a way of dividing some set into disjoint subsets. More interestingly for us, the partitioning of a set can be carried out by an equivalence relation, which is a reflexive, symmetric and transitive relation. If $R$ is an equivalence relation, $[x]_{R}$ represents the equivalence class containing $x$. An equivalence class simply collects in a set all the elements that are equal with respect to some equivalence relation. Thus, if $y$ is also a member of $[x]_{R}$, then $[x]_{R}=[y]_{R}$. Each subset that is a member of some partition is called a cell. An equivalence relation $R$ is able to induce a partition on a set $A$, because any two members $x$ and $y$ can only be in the same cell if (and only if) they are related by $R$.

a. Partition: Let $A$ be a non-empty set. A partition is a collection of subsets of $A$ iff ( $i$ ) for any two subsets $X$ and $Y, X \cap Y=\varnothing$ and (ii) the union of all subsets of $A$ equals $A$.

b. Equivalence Relation: Let $R$ be an equivalence relation. Then: $a \simeq_{R} b$ iff $\forall x[(R(a, x) \leftrightarrow R(b, x)) \wedge(R(x, a) \leftrightarrow R(x, b))]$

c. Equivalence Class: Let [ $]_{R}$ be a function from a domain $D$ to $P O W(D)$ such that: $\forall x \in D\left[[x]_{R}=\left\{y: y \in D \wedge x \simeq_{R} y\right\}\right]$

Let us return to Fido in (23). Given the equivalence relation be the same breed as, Fido is a member of the cell containing border collies, the equivalence class $[F]_{\text {breed }}$. By the same token, 
if the equivalence relation were have the same role as, Fido would be in the cell containing watch dogs, $[F]_{\text {role }}$. Given the properties of partitions, Fido cannot live in two cells at the same time, and so we have to chose one or the other equivalence relation,. Hence the ill-formedness of (23).

We can now look into how to extend the partition talk to degrees. I follow Cresswell (1976) in assuming that degrees can be viewed as equivalence classes of individuals (see Klein, 1980; Rullmann, 1995). I illustrate the main idea with an adjective $A$. Associated with any gradable predicate (an adjective, adverb, verb, etc.) there is a two-place relation $\geq_{A}$, and a set $D_{A}$. The set $D_{A}$ is a subset of the universe of discourse containing all and only those objects of which the adjective can be sensibly predicated. This is just a lexical requirement to make sure that a set like $D_{\text {tall }}$ contains people, mountains, etc., but not ideas or colors, since the latter cannot be sensibly attributed a height. The relation $\geq_{A}$ is reflective of our conceptual ability to determine, from any two individuals, which has more of a certain quality than another. Cresswell (1976) suggested to define $\geq_{A}$ as follows:

$$
\left\langle D_{\text {tall }},\left\{\langle x, y\rangle: x, y \in D_{\text {tall }} \text { and } x \text { is as tall as } y\right\}\right\rangle
$$

From here the relation $\geq_{A}$ may induce a totally-ordered relation $\geq_{A}$ on the members of $D E G_{A}$ such that $\operatorname{deg}_{A}(x) \geq_{A} d e g_{A}(y)$ iff $x \geq_{A} y \wedge y \Varangle_{A} x$. In this case, we can easily define an equivalence relation from $\geq_{A}$, and partition a domain according to the resulting equivalence relation, where the degree of $A$-ness of an object $x$, say $\operatorname{deg}_{A}(x)$ is defined as the set of all objects that stand in the $\simeq_{A}$ relation to $x$ :

$$
\begin{array}{ll}
\text { a. } & x \simeq_{A} y \leftrightarrow x \geq_{A} y \wedge y \geq_{A} x \\
\text { b. } & \operatorname{deg} g_{A}(x)=\left\{y \in D_{A}: x \simeq_{A} y\right\}
\end{array}
$$

As a consequence, the degree to which Liz is tall, $\operatorname{deg}_{\text {tall }}(\mathrm{Liz})$ can now be identified with the set of all objects that are exactly as tall as Liz. In this view, each degree $d$ corresponds to one of the cells in the partition $D E G_{A}$ induced on the set $D_{A}$. For instance, in the case of $D E G_{\text {tall }}$ (and a very reduced domain) we may have: ${ }^{5}$

$$
\begin{array}{|l|l|l|l|}
\hline d_{5.8 f}: \text { John, Sue Liz } & d_{5.9 f}: \text { Mary, Al } & d_{6 f}: \text { Mike, Helen } & d_{6.1 f} \text { : Hilary } \\
\hline
\end{array}
$$

Establishing partitions from pluralities works exactly the same. We only have to be careful to avoid overlapping individuals. That is, we must avoid that a plurality of two individuals $a \oplus b$, living in the cell corresponding to those pluralities of cardinality 2 , be also members of some other cell in the partition. For instance, one could expect that if $a \oplus b$ teams up with individual $c$ to form the plurality $a \oplus b \oplus c$, then individuals $a$ and $b$ would simultaneously be in the cell corresponding to pluralities of cardinality 3 as well. The solution is to adopt a Link (1983) style approach to pluralities, where plural entities are just sums of individuals (and not sets), as concrete as the individuals that serve to define them and of the same logical

\footnotetext{
${ }^{5}$ Notice that the thresholds of the degrees should be overtly determined, so that there is no vagueness whatsoever as to where exactly every individual belongs in the partition. In the example above the cut-off point was the nearest inch, so the actual equivalence relation should read be as tall as, to the nearest inch.
} 
type, creating a non-monotone domain of individuals. If a mapping exists between degrees and sets of individuals, as discussed above, each level in a Linkian structure can be seen as an equivalence class. Assuming that cardinalities are simply degrees, as it is common practice, we can create a partition $D E G_{\text {card }}$ on $D$ by the equivalence relation $\simeq_{\text {card }}$.

$$
\begin{aligned}
& \text { a. } \quad x \simeq_{\text {card }} y \leftrightarrow x \geq_{\text {card }} y \wedge y \geq_{\text {card }} x \\
& \text { b. } \quad d e g_{\text {card }}(x)=\left\{y \in{ }^{*} D: x \simeq_{A} y\right\}
\end{aligned}
$$

$$
\text { [where } \geq_{\text {card }}=\text { a cardinality as big as] }
$$

The result is a partition of the domain of plural individuals according to their cardinality. For instance, the equivalence class $[a \oplus b]_{\text {card }}$ corresponds to all plural individuals of cardinality 2 in the domain, such that $[a \oplus b]_{c a r d}=[a \oplus c]_{\text {card }}=[b \oplus c]_{\text {card }}$, etc. Because plural individuals are individuals with full rights, we need not look into their composing parts. That is, that $a$ and $b$ belong to one cell and $a \oplus b$ to a different cell is inconsequential in this respect.

\subsection{Connecting the dots}

In order to make the connection between ad hoc subkinds and amount interpretations of relative clauses explicit, we have to look a bit further into ad hoc subkinds. Ad hoc subkinds are inherently vague referring expressions. Although they refer to subkinds, they do not do so in a direct way. Compare:

a. The blue whale is becoming extinct.

b. The whales that you like so much are becoming extinct.

One can refer to a subkind by directly mentioning its name. In this case, blue whale stands for a (taxonomic) subkind of whale. But very few subkinds have names. For all we know, the kind of whales that you like so much could be blue whales, but it could as well be almost any collection of whales that you fancy. That is, the subkind whales that you like so much are a subkind just by virtue of your liking them so much. In this case, then, the only "sufficiently regular behavior" that we may impute them is precisely that you like them so much.

I suggest that the sole role of the relative clause in ad hoc subkind reference constructions is to provide information that helps determine what the relevant sufficiently regular behavior is. How exactly does the relative clause fulfill this role? It does so by restricting, in more or less the usual way, the denotation of the kind-denoting NP, e.g. whale in (30b), to a subset of whales. Crucially, this subset must be a member of one cell in a partition of whale subkinds. Given the nature of partitions, information about one cell can help us fill in the rest of the cells; for instance, by lumping together in one cell the individual whales that you like, and all the ones that do not belong in this cell occupying the sole other cell of the partition. The more information we might have about your preferences, the richer the partition could be. Under this view, a critical part of resolving ad hoc subkind reference is being able to determine an equivalence relation that puts all the whales that you like in a single cell. 
This is not always as straightforward as it may seem and, oftentimes, vagueness is rampant. In (1), the champagne that we spilled last night is referring to an ad hoc subkind of champagne. If we go with the taxonomic interpretation of the sentence, we partition the domain of champagne into its different subkinds, and we assume that the particular champagne that the spilled last night lives in one of the cells. For instance, if they spilled a very rare kind of prestige cuvée, we would require a partition based on taxonomic kinds.

$$
\begin{array}{|l|l|l|l|}
\hline \text { Prest. cuvée } & \text { Blanc de noirs } & \text { Blanc de blancs } & \text { Rosé } \\
\hline
\end{array}
$$

Each one of the cells above contains the individual instances of champagne that correspond to each kind. ${ }^{6}$ Carlson's (1977a) disjointness condition is met by resorting to an equivalence relation like be the same kind as. Now, it could be that the reason why it would take us so long to find the champagne that we spilled last night is because it was much sweater than usual. In that case, we can generate the relevant partition by sweetness-gr. of sugar per liter-from an equivalence relation like be as sweet as.

$$
\begin{array}{|l|l||l|l|l|}
\hline d<6 & 12.1<d<17 & 17.1<d<32 & 32.1<d<50 & \ldots<d<\ldots \\
\hline
\end{array}
$$

To reiterate: subkind reference must be mediated by a partition to ensure that the domain is covered by non-overlapping sets. This partitioning is carried out by an equivalence relation that is only contextually determined. As long as this is observed, any equivalence relation might do. Thus, the only difference between (31) and (32) above is that different equivalence relations are picked in different contexts. At this point, it is straightforward to extend the same reasoning to the classical AR examples and amount interpretations. Since we know that cardinalities can be defined in terms of equivalence classes, there is no reason why the required equivalence relation cannot be of the form be as much as. Thus, for the classical champagne example in (1), we could envision a partition by volume, as in (33).

$$
\begin{array}{|l|l|l|l|l|l|}
\hline 0 \mathrm{~L} \leq d<1 \mathrm{~L} & 1.1 \mathrm{~L}<d<2 \mathrm{~L} & 2.1 \mathrm{~L}<d<3 \mathrm{~L} & 3.1 \mathrm{~L}<d<4 \mathrm{~L} & 4.1 \mathrm{~L}<d<5 \mathrm{~L} & \ldots<d<\ldots \\
\hline
\end{array}
$$

If this rationale is correct, amount interpretations of relative clauses are simply a case of $a d$ hoc subkind reference. Thus, the only analysis we need is one that derives ad hoc subkind reference, and no appeal to degree semantics is necessary.

To sum up, making reference to subkinds requires structuring the domain in a certain way. I argued that one way of capturing this requirement is by partitioning the relevant domain. Once this step is taken, a parsimonious account of amount interpretations of relative clauses is made available. Because degrees are definable in terms of equivalence classes, we can exploit the independently-needed mechanisms of $a d$ hoc kind reference and induce a partition that gives us equivalence classes of quantities or amounts. Given the evidence in sections $\S 2$ through $\S 3$, this account offers a number of advantages: $(i)$ it accounts for the pervasive similarities between kind and amount referring relative clauses in terms of their the syntactic/semantic properties, (ii) it accounts for the lack of evidence for degree abstraction, and (iii) it relies on mechanisms

\footnotetext{
${ }^{6}$ In this respect, the table above is just a shortcut to the actual partition, whose members are always individuals, not kinds.
} 
that are independently needed for the interpretation of subkind-referring expressions, as well as extant analyses of degrees as equivalence classes.

\section{Compositional implementation}

In laying out my assumptions about kind reference I am following Chierchia (1998) for the most part. Kinds are individuals whose spatiotemporal manifestations are discontinuous. In this sense, the kind DOG can be identified as the sum of all individual dogs, which can then be modeled as the largest member of the plural individual comprising all dogs. For the majority of properties, like the property of being a dog, there is a corresponding kind, the dog-kind. Conversely, natural kinds have a corresponding property (the property of belonging to that kind). Properties may be systematically mapped to their individual correlates via a nominalization function, the "down" operator $\cap$. Likewise, individual kinds may be mapped to their corresponding properties via the inverse of $\cap$, the "up" operator $\cup$.

a. Predicativization: Let $d$ be a kind. Then for any world/situation $s, \cup d=\lambda x . \leq d_{s}$, if $d$ is defined, false otherwise (where $d_{s}$ is the plural individual that comprises all of the atomic members of the kind).

b. Nominalization: For any property $P$ and world/situation $s, \cap P=\lambda s . \imath P_{s}$ if $\lambda s . \imath P_{s}$ is in $K$; else undefined (where $P_{S}$ is the extension of $P$ in $s$ and $K$ is the set of kinds).

Kinds are individuals with their own rights, and so they belong to their own domain $D_{k}$, a subset of $D .^{7}$ Thus, we can talk about the domain of object-individuals $D_{o}$, to the exclusion of the domain of kind individuals, $D_{k}$. Kinds have the possibility to combine both with kind-level and with object-level predicates. In the first case, kinds are attributed some property directly by the main predicate, (35a). In the second case, most commonly with episodic sentences, we encounter a mismatch between a kind denoting argument and a predicate that lexically selects for non-kind predicates, (35b).

a. Dogs are $\{$ widespread/extinct/common $\}$.

b. Dogs are barking outside my window.

Example (35a) is a case of direct-kind reference: to derive this interpretation, we simply apply the kind denoting term to the predicate, e.g. extinct (DOG). The example in (35b) is different in that the dog-kind now serves as an argument to an individual-selecting predicate. In this case, the predicate does not attribute properties to the dog-kind, but to object-level instances of the dog-kind; (35b) asserts the existence of some individual dog that is barking. To achieve this result, Chierchia (1998) proposes a new rule of composition, Derived Kind Predication (DKP henceforth), that solves two problems: it provides a means to solve the sortal mismatch and introduces existential quantification over instances of a kind.

\footnotetext{
${ }^{7}$ In order to represent kinds and object variables, I follow the convention of using the subscripts $k$ for kind-level and $o$ for object-level variables.
} 


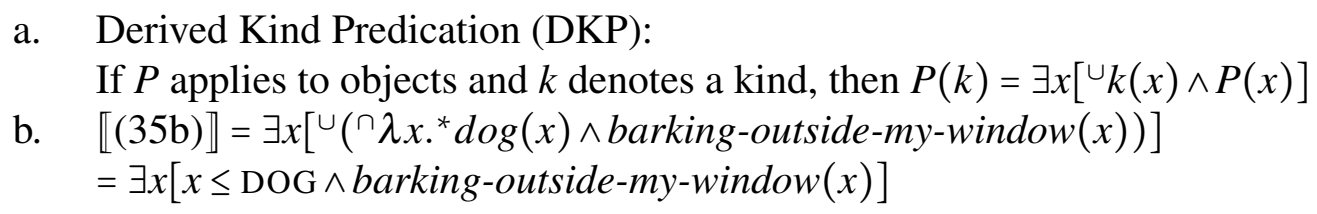

The next step in arriving at the desired ad hoc subkind interpretations involves a mapping from kinds to subkinds. ${ }^{8}$ In accordance with the discussion above, however, we need a mapping that will partition kinds, not just any subkind extracting operation. The partition function below meets these two criteria (cf. Gillon, 1987; Schwarzschild, 1996): a partition of a kind $K$ is a set of subsets of ${ }^{\cup} K$ that covers ${ }^{\cup} K$ and whose members do not share any instantiating individuals.

Partition function: A partition function $\Pi$ is a $\langle k, k t\rangle$ function such that for any kind $K, \Pi(K)$ meets two conditions:

a. Cover: $\forall x_{o}\left[x_{o} \leq K \rightarrow \exists y_{k} \in \Pi(K)\left[x_{o} \leq y_{k}\right]\right]$

b. No overlap: $\forall x_{o}\left[\exists y_{k} \in \prod(K)\left[x_{o} \leq y_{k}\right] \rightarrow \neg \exists z_{k} \in \Pi(K)\left[y_{k} \neq z_{k} \wedge x_{o} \leq z_{k}\right]\right]$

As an illustration, consider the case of $K=$ DOG, where we partition the dog-kind taxonomically (i.e. $\Pi($ DOG $)=\{$ COLlie, PUG, GREYhound, BEAGLE, ... $\}$ ). Then condition (a) states that if $x_{o}$ is an instance of the kind DOG, there is some subkind $y_{k}$ in the set of subkinds $П$ (DOG) that $x_{o}$ is also an instance of. This condition makes sure that all particular dogs belong to some subkind, to some breed in this case. In turn, condition (b) states that if $x_{o}$ is an instance of the subkind $y_{k}$, there will be no additional subkind $z_{k}$ in $\Pi(\mathrm{DOG})$ such that $x_{o}$ also realizes. This is reflective of the fact that, if Fido is a beagle, he cannot be any other breed. More generally, the function ensures that if we partition the dog-kinds by breed, all border-collies will be in the same cell of the partition, and, say watch-dog border-collies will not be able to occupy their own-despite being a subkind of dogs as well in the actual world.

We can now use the partition function in (37) to provide a compositional account of ad hoc kind-referring terms. First, a kind must be partitioned into a set of individual correlates of its subkinds. We can do this by defining a kind-to-subkind operator that employs the partition function (cf. Zamparelli, 1998). Call this operator $\kappa$.

$$
\llbracket \kappa \rrbracket=\lambda x_{k} \cdot \lambda y_{k} \cdot \Pi\left(x_{k}\right)\left(y_{k}\right)
$$

From a semantic standpoint, we can think of $\kappa$ as doing covertly the task that the noun kind does overtly. It targets a kind $x_{k}$ and returns a set of kind-individuals that partitions $x_{k}$. The function returns the set of (individual correlates of) subkinds that are in the partition.

$$
\llbracket \kappa \rrbracket(\llbracket \mathrm{DOG} \rrbracket)=\lambda y_{k} \cdot \Pi(\mathrm{DOG})\left(y_{k}\right)=\{\text { GREYHOUND, BORDER COLLIE, BEAGLE }, \ldots\}
$$

In this case, we have partitioned the domain of DOG subkinds according to their taxonomy, making sure on the way that no one dog belongs to two separate kinds. So far we have suc-

\footnotetext{
${ }^{8}$ There are a number of mappings in the literature between kinds and subkinds (e.g. Krifka et al., 1995; Wilkinson, 1995; Zamparelli, 1998), usually carried out by an operator, whose meaning is generally taken to be very similar to the noun kind in expressions like kind of dog.
} 
cessfully reproduced Carlson's (1977a) results, but we have not quite achieved our goal of accounting for ad hoc kind reference. As I suggested earlier, two pieces of information are required in order to form an ad hoc kind in real time: $(i)$ a semantic sortal-something to be a kind of-, and (ii) some means to identify what the relevant subkind is, i.e. to identify its sufficiently regular behavior. This is shown by the contrast between the two sentences in (40):

a. That kind of dog is dangerous.

b. *The kind of dog is dangerous.

In the two cases in (40) the semantic sortal is provided by the kind-referring noun $d o g$, but only (40a) provides a means to identify the relevant properties of the dogs that are to be recognized as dog-subkinds; in this case it does so by anaphorically referring to it. The variant in (40b) lacks this second piece of information and reference to a kind fails.

A similar state of affairs holds in the absence of the noun kind. When no natural kind nor an antecedent for the intended subkind is available, we can use the NP Rel Clause constructions to refer to ad hoc kinds. This is because the relative clause itself can express a regularity that characterizes the kind in question, thus aiding in kind reference resolution. With kind-referring terms involving the noun kind, the role of the relative clause is obvious. But given our analysis of the $\kappa$-operator, the role of the relative clause in ad hoc kind reference without the noun kind should follow analogously.

In order to capture this difference formally we can think of the relative clause as a means to further narrow the kind-referring potential of kind-referring NPs. It is in this respect that appealing to partitions becomes specially useful. We can easily modify $\kappa$ so that it makes reference to an additional argument, a predicate $P$, and states a new condition whereby objects in the intersection of $P$ and the property correlate of the kind $K$ all live in the same cell of some partition of $K$. This can be done as follows. Consider first a revised version of $\kappa, \kappa+$.

$$
\llbracket \kappa+\rrbracket=\lambda x_{k} \cdot \lambda P_{\langle e t\rangle} \cdot \lambda y_{k} \cdot \Pi\left(x_{y}\right)\left(y_{k}\right) \wedge \forall z_{o}\left[z_{o} \leq x_{k} \wedge P\left(z_{o}\right) \rightarrow z_{o} \leq y_{k}\right]
$$

After applying to an individual kind $x_{k}$ and a property $P$ of individuals, $\kappa+$ returns the subkinds that include objects whose realizations are both instances of $x_{k}$ and members of $P$. The task of $P$, the relative clause, is to provide information about the regular behavior that we must impute to the subkind in question. This is achieved by letting the relative clause do its usual job and interpreting it intersectively.

Let us work out a concrete example, the lions that eat people, from (22c). The term lions that eat people refers to a kind, but not to a natural or well-established one, so this is a task for $\kappa+$. For concreteness, assume a syntactic structure along the lines in (42). By the time $\kappa+$ gets to enter into the derivation, the NP already denotes a kind. ${ }^{9}$

\footnotetext{
${ }^{9}$ There a number of ways of doing this (see e.g. Carlson, 1977b; Zamparelli, 1998; Dayal, 2004; Kratzer, 2005). Bear in mind however that different options entail different views of how nouns come to denote kinds. At any rate, this is a simplifying assumption, and nothing about how ad hoc kind-referring terms are derived hinges on this decision.
} 
$\left[{ }_{D P}[D\right.$ the $]\left[{ }_{N P 1}\left[\mathrm{NP} 2 \kappa+[\mathrm{NP} 3 \text { lion }]_{i}\right]\left[{ }_{\mathrm{CP}}\right.\right.$ that $\mathrm{t}_{i}$ eat people $\left.\left.]\right]\right]$

$$
\begin{array}{ll}
\text { a. } & \llbracket \mathrm{NP} 2 \rrbracket=\llbracket \kappa+\rrbracket(\llbracket \mathrm{LION} \rrbracket) \\
& =\lambda P_{\langle e t\rangle} \cdot \lambda y_{k} \cdot \prod(\mathrm{LION})\left(y_{k}\right) \wedge \forall z_{o}\left[z_{o} \leq \mathrm{LION} \wedge P\left(z_{o}\right) \rightarrow z_{o} \leq y_{k}\right] \\
\text { b. } & \llbracket \mathrm{NP} 1 \rrbracket=\lambda y_{k} \cdot \prod(\mathrm{LION})\left(y_{k}\right) \wedge \forall z_{o}\left[z_{o} \leq \mathrm{LION} \wedge \operatorname{eat}-\operatorname{people}\left(z_{o}\right) \rightarrow z_{o} \leq y_{k}\right]
\end{array}
$$

The last line above returns a set of subkinds of the lion-kind that partitions the domain of lions and where the all the object-level lions of which $P$ holds constitute an instance of one such kind. This is still too weak a meaning. But now the definite article can simply contribute an $l$ operator: $\llbracket$ the $P \rrbracket=$ the contextually salient largest member of $P$, if there is one, else undefined. The article applies to the set of subkinds of lions denoted by NP1 and returns the single salient subkind of which all the people-eating lions are an instance, i.e. the individual correlate of the property be a people-eating lion.

$$
\begin{aligned}
& \imath y_{k} \cdot \prod(\mathrm{LION})\left(y_{k}\right) \wedge \forall z_{o}\left[z_{o} \leq \mathrm{LION} \wedge \text { eat-people }\left(z_{o}\right) \rightarrow z_{o} \leq y_{k}\right] \\
& =\cap\left(\lambda z .{ }^{*} \operatorname{lion}(z) \wedge \text { eat people }(z)\right)
\end{aligned}
$$

As a consequence, non-people-eating lions and lions that eat other things besides people will have to live in other cells of the partition. ${ }^{10}$ It follows, then, that the cells in the partition cannot contain taxonomic subkinds anymore, since no partition of lions in terms of their subspecies will contain the ad hoc subkind of lions that eat people in one its cells. Thus, as desired, this method of referencing ad hoc subkinds overrides any other natural ways of picking the relevant subkinds (e.g. taxonomic properties, etc.). The most likely way to complete the rest of the partition is to find a suitable equivalence relation that groups all people-eating lions in the same cell. An equivalence relation eat the same as might do. With this equivalence relation we may obtain a partition of the lion-kind like the following.

\section{\{LIONS THAT EAT PEOPLE, LIONS THAT EAT ZEBRAS, LIONS THAT EAT CARRION...}

What matters most is that the modifier, the relative clause in this case, is informing us about what one of the subkinds must look like. The resulting DP can serve as an argument to kindlevel predicates in the usual way. Alternatively, it can serve as non-kind-selecting predicates via Derived Kind Predication (see (36a) above): a sentence like (46a) asserts the existence of an instantiation of the ad hoc eating-people-lion-kind, and that you like (some of) those instantiations.

a. You like the lions that eat people.

b. $\quad \llbracket(46 \mathrm{a}) \rrbracket=\exists y\left[\cup\left(\cap \lambda z .{ }^{*} \operatorname{lion}(z) \wedge\right.\right.$ eat-people $\left.(z)\right)(y) \wedge \operatorname{like}(y)($ you $\left.)\right]$ $=\exists y[y \leq \operatorname{LION} \wedge$ eat-people $(y) \wedge \operatorname{like}($ you, $y)]$

Notice that, practically speaking, (46a) may be interpreted in a number of ways. This is because the semantics of $\kappa+$ only forces us to find a partition of lions where the lions that eat people live in one cell, but it does not force us to talk about the fact that these lions eat people. As

\footnotetext{
${ }^{10}$ As mentioned above, in order to build the partition properly the relevant description should be explicit enough to avoid overlap. Thus, we should have $\{$ LIONS THAT EAT ONLY PEOPLE, LIONS THAT EAT ONLY ZEBRAS, ...
} 
with ordinary kind predication, there might be a number of reasons to refer to a kind. Thus, the traits of the lions that you like in (46a) need not be determined by the relative clause. For instance, it could be that lions that eat people have a number of associated characteristics (e.g. they are faster, smarter, etc.) that you like, despite the fact that you are not fond of their habit to eat people. In such case, (46a) is true and felicitous, as captured by (46b).

\section{Conclusions}

The merits of looking at English so-called ARs as a species of kind-referring relative clauses are various. All the properties of amount interpretations of relative clauses discussed in sections $\S 2$ through $\S 3$ follow without additional stipulations, namely, $(i)$ it accounts for the AMOUNT $\subseteq$ KIND generalization in (4) above, which states that amount interpretations of relative clauses are parasitic on kind interpretations and (ii) it explains why amount interpretations are not subject to the typical restrictions that we observe with constructions that involve degree-abstraction and degree-operators. Thus, if the results reported here are on the right track, the relative clauses discussed in this paper are not "Amount Relatives", literally speaking, but ad hoc kind-referring expressions.

\section{References}

Carlson, G. (1977a). Amount relatives. Language 53, 520-542.

Carlson, G. (1977b). Reference to kinds in English. Ph. D. thesis, UMass Amherst.

Chierchia, G. (1998). Reference to kinds across languages. Natural Language Semantics 6(4), 339-405.

Cresswell, M. J. (1976). The semantics of degree. In B. H. Partee (Ed.), Montague grammar, pp. 261-292. New York: Academic Press.

Dayal, V. (2004). Number marking and (in)definiteness in kind terms. Linguistics and Philosophy 27(4), 393-450.

Gillon, B. S. (1987). The readings of plural noun phrases in English. Linguistics and Philosophy 10(2), 199-219.

Grosu, A. and F. Landman (1998). Strange relatives of the third kind. Natural Language Semantics 6(2), 125-170.

Grosu, A. and F. Landman (2017). Amount relatives. To appear in M. Everaert and H. van Riemsdijk (eds.), The Companion to Syntax, 2nd Ed., Ch. 7; Wiley-Blackwell, Oxford.

Heim, I. (1987). Where does the definiteness restriction apply. evidence from the definiteness of variables. In E. Reuland and A. ter Meulen (Eds.), The Representation of (In)definiteness, pp. 21-42. MIT Press.

Herdan, S. (2008). Degrees and Amounts in Relative Clauses. Ph. D. thesis, UConn.

Kennedy, C. (1999). Projecting the Adjective: The Syntax and Semantics of Gradability and Comparison. New York: Garland.

Klein, E. (1980). A semantics for positive and comparative adjectives. Linguistics and Philosophy 4(1), 1-45.

Kratzer, A. (2005). Indefinites and the operators they depend on: From Japanese to Salish. In G. N. Carlson and F. J. Pelletier (Eds.), Reference and Quantification: The Partee Effect, pp. 113-142. CSLI Publications. 
Krifka, M., F. J. Pelletier, G. N. Carlson, A. ter Meulen, G. Chierchia, and G. Link (1995). Introduction to genericity. In G. N. Carlson and F. J. Pelletier (Eds.), The Generic Book, pp. 1-1124. Chicago: CUP.

Lechner, W. (2001). Reduced and phrasal comparatives. Natural Language and Linguistic Theory 19(4), 683-735.

Link, G. (1983). The logical analysis of plurals and mass terms. In R. Bauerle, C. Schwarze, and A. von Stechow (Eds.), Meaning, use and interpretation of language, pp. 302-323. De Gruyter.

McNally, L. (2008). DP-internal only, amount relatives and relatives out of existentials. Linguistic Inquiry 39(1), 161-169.

Meier, C. (2015). Amount relatives as generalized quantifiers. Ms., Goethe University.

Partee, B. H. (1973). Some transformational extensions of Montague Grammar. Journal of Philosophical Logic 2, 509-534.

Quine, W. V. O. (1960). Word and Object. Cambridge: MIT Press.

Rullmann, H. (1995). Maximality in the semantics of wh-constructions. Ph. D. thesis, UMass Amherst.

Schwarzschild, R. (1996). Pluralities. Kluwer, Dordrecht.

von Fintel, K. (1999). Amount relatives and the meaning of chains. Ms., MIT.

von Stechow, A. (1984). Comparing semantic theories of comparison. Journal of Semantics 3(1-2), 1-77.

Wilkinson, K. (1995). The semantics of the common noun kind. In N. Carlson, Gregory and F. J. Pelletier (Eds.), The generic book, pp. 383-397. Chicago: CUP.

Zamparelli, R. (1998). A theory of kinds, partitives and $o f / z$ possessives. In A. Alexiadou and C. Wilder (Eds.), Possessors, predicates and movement in the determiner phrase, pp. 259-304. Amsterdam: John Benjamins Publishing Company. 